\title{
Epithelioid Myoepithelioma of the Accessory Parotid Gland: Pathological and Magnetic Resonance Imaging Findings
}

\author{
Hiroyoshi Iguchi $^{\mathrm{a}}$ Kei Yamada ${ }^{\mathrm{a}}$ Hideo Yamane $^{\mathrm{a}}$ Shigeo Hashimoto ${ }^{\mathrm{b}}$ \\ ${ }^{a}$ Department of Otolaryngology and Head and Neck Surgery, Osaka City University \\ Graduate School of Medicine, and ${ }^{b}$ Department of Diagnostic Pathology, PL General \\ Hospital, Osaka, Japan
}

\section{Key Words}

Epithelioid myoepithelioma - Accessory parotid gland · Salivary gland · Magnetic resonance imaging · Surgery

\begin{abstract}
Tumors of the accessory parotid gland (APG) are rare, and pleomorphic adenoma (PA) is the most common benign APG tumor subtype. Myoepithelioma of the APG is much rarer than $\mathrm{PA}$, and to date, only 5 cases have been sporadically reported in the English literature. We describe the clinicopathological and MRI findings of an epithelioid myoepithelioma of the APG that was treated in our hospital. The patient's only clinical symptom was a slow-growing and painless mid-cheek mass. The tumor was suspected to be PA before surgery based on the following MRI findings: (1) a well-circumscribed and lobulated contour, (2) isointensity and hyperintensity relative to the muscle on T1- and T2-weighted images (WIs), respectively, (3) good enhancement on contrast-enhanced T1-WIs, (4) peripheral hypointensity on T2-WIs, and (5) a gradual time-signal intensity curve enhancement pattern on gadolinium-enhanced dynamic MRI. The tumor was completely resected via a standard parotidectomy approach, and the postoperative pathological examination of the tumor, including immunohistochemistry, confirmed the diagnosis of epithelioid myoepithelioma. As it is hardly possible to distinguish myoepithelioma from PA and low-grade malignant tumors preoperatively, a pathological examination using frozen sections is helpful for surgical strategy-related decisions.


Iguchi et al.: Epithelioid Myoepithelioma of the Accessory Parotid Gland: Pathological and Magnetic Resonance Imaging Findings

\section{Introduction}

Myoepithelioma is a benign tumor that can originate from any secretory system throughout the body. In the salivary gland, myoepithelioma is rare, accounting for $1 \%$ of all salivary gland tumors [1]. Although myoepithelioma of the salivary gland originates most frequently in the parotid gland [2], myoepithelioma of the accessory parotid gland (APG) is extremely rare, and to date, only 5 cases have been reported in the English literature [2-5]. Because the clinicopathological and MRI findings of myoepithelioma of the APG have not yet been fully described, and given the rarity of this condition, we hereby describe the clinicopathological and MRI findings of an epithelioid myoepithelioma of the APG that was treated in our hospital.

\section{Case Report}

A 31-year-old woman presented with a 5-year history of a slowly enlarging left midcheek mass in the pre-parotid region. Upon examination, a mobile non-tender and smoothsurfaced mass measuring $20 \mathrm{~mm}$ in the greatest dimension was noted in the left pre-parotid region. Her facial nerve function was intact. The serum amylase level was within the normal range. An unenhanced CT of the neck confirmed a well-circumscribed and homogenous mass measuring $18 \times 10 \mathrm{~mm}$ and located anterior to the left main parotid gland and on the surface of the masseter muscle. This lobulated mass demonstrated isointensity and hyperintensity relative to the muscle on T1- and T2-weighted MRI images (WIs), respectively, and homogeneous enhancement on contrast-enhanced T1-WIs (fig. 1a-c). A time-signal intensity curve (TIC) generated from a dynamic MRI study revealed a gradual enhancement pattern (fig. 1d). On T2-WIs, the mass periphery was surrounded by hypointensity that was suggestive of a fibrous capsule. The tumor was most likely considered a pleomorphic adenoma (PA), given the clinical presentation and MRI findings. No remarkable cervical lymphadenopathy was detected. The patient refused fine-needle aspiration cytology (FNAC). The mass was resected under general anesthesia via a standard parotidectomy incision. The mass and the surrounding salivary tissue were completely separated from the main parotid gland and connected to the parotid duct via a ductule. Myoepithelioma was suspected during an intraoperative pathological examination of a frozen specimen. All facial nerve branches and the parotid duct were successfully preserved, and postoperative complications such as facial paralysis and salivary fistula were not observed. Grossly, the tumor was well encapsulated, solid, and lobular (fig. 2a), with a yellowish-white cut surface. Microscopically, the tumor primarily comprised cells with round nuclei and a few cells with oval and slightly spindle-shaped nuclei. The neoplastic cells were arranged in an epithelial and mostly latticelike structure with hyalinous and mucinous stroma. Neither cartilaginous myxoid stroma nor a glandular structure was observed (fig. 2b). Immunohistochemical staining indicated a positivity for calponin (fig. 2c), CAM5.2 (cytokeratin stain), S-100 protein, smooth muscle actin (SMA), cytokeratin14, and AE1/AE3 (pan-keratin stain), as well as negativity for carcinoembryonic antigen. Based on these results, the final histopathological diagnosis of the tumor was epithelioid myoepithelioma. There have been no signs of recurrence at 3.5 years after surgery. 
Iguchi et al.: Epithelioid Myoepithelioma of the Accessory Parotid Gland: Pathological and Magnetic Resonance Imaging Findings

\section{Discussion}

PA is reportedly the most common subtype of benign APG tumors; in contrast, myoepithelioma represents an extremely rare APG tumor pathology. Only 5 cases of myoepithelioma of the APG have been reported in the English literature. Kawashima et al. [4] reported a case in a 34-year-old woman, Isogai et al. [2] reported a case of plasmacytoid myoepithelioma of the APG in a 47-year-old woman, and Sun et al. [5] reported a case in a 41-year-old man and one in a 22-year-old woman; all of these cases were treated by surgery. Recently, Xie et al. [3] described the fifth case in a 65-year-old man who was treated via an endoscopic approach.

There have been only a few reports on the imaging findings of myoepithelioma of the parotid gland. According to Wang et al. [6], myoepithelioma of the parotid gland is characterized by a well-defined and smooth or lobulated mass with homogeneous or inhomogeneous enhancement on the CT. Although MRI is a widely used modality for evaluating parotid gland tumors because it provides a greater amount of morphological information than CT does, the MRI features of myoepithelioma of the APG have not been previously described. The typical MRI findings of PA, in contrast, have been well described to include a lobulated or ovalshaped well-defined encapsulated mass, a mass with a hypointensity on T1-WIs and hyperintensity surrounded by a hypointense capsule on T2-WIs [7] as well as a gradual TIC enhancement pattern in dynamic MRI studies [8]. In the present case, the myoepithelioma of the APG was isointense relative to the muscle on T1-WIs, hyperintense relative to the muscle on T2-WIs, and well enhanced on contrast-enhanced T1-WIs. Peripheral hypointensity on T2-WIs was identified and suggested a fibrous capsule. Additionally, a TIC generated from a gadolinium-enhanced dynamic MRI study displayed a gradual enhancement pattern. We preoperatively suspected the mass to be PA because the MRI findings were completely consistent with those of PA.

As the name indicates, myoepithelioma comprises neoplastic cells exhibiting myoepithelial differentiation; these cells display various morphologic patterns such as spindle, plasmacytoid, epithelioid, and clear types. Because of this morphologic diversity, it is usually difficult to definitively diagnose myoepithelioma by FNAC before surgery [9], although Das et al. [10] reported a case of a successful preoperative parotid myoepithelioma diagnosis via a cytological examination and immunohistochemistry. The main differential diagnoses of myoepithelioma include PA, adenoid cystic carcinoma, other salivary tumors without obvious ductal differentiation, and mesenchymal tumors. In particular, we should always consider various low-grade malignant tumors when interpreting the FNAC results from salivary tumors [10]. A histopathological examination of the resected specimen can yield a definitive diagnosis of myoepithelioma, and immunohistochemical staining plays an important role in the detection of neoplastic myoepithelial cells. Such cells are typically positive for cytokeratin, vimentin, S-100 protein, calponin, SMA, and glial fibrillary acidic protein [10], but negative for carcinoembryonic antigen. In our case, the histopathological findings of an epithelial and lattice-like arrangement of neoplastic cells with hyalinous and mucinous stroma were also characteristic components of PA. However, neither cartilaginous myxoid stroma nor a glandular structure was found in our case, and the tumor was entirely encapsulated by connective tissue. Additionally, positive immunohistochemical staining of the neoplastic cells for CAM5.2, cytokeratin14, AE1/AE3, SMA, and calponin indicated that these neoplastic cells possessed both epithelial and myoepithelial characters. These histopathological findings resulted in the diagnosis of epithelioid myoepithelioma. 
Iguchi et al.: Epithelioid Myoepithelioma of the Accessory Parotid Gland: Pathological and Magnetic Resonance Imaging Findings

The malignant transformation of epithelioid myoepithelioma has been described previously [2]. Complete resection along with the surrounding salivary tissue is recommended for myoepithelioma management in order to avoid tumor recurrence.

In conclusion, a mid-cheek mass could easily be suspected as an APG tumor according to the clinical presentation and imaging findings. However, because myoepithelioma is an extremely rare APG tumor subtype and the imaging findings of myoepithelioma resemble those of PA, which is the most common benign APG tumor subtype, we believe that it is nearly impossible to preoperatively distinguish myoepithelioma of the APG from PA of the APG or low-grade malignant tumors, even by using FNAC, given that Kawashima et al. [4] also misdiagnosed a myoepithelioma of the APG as a suspected adenoid cystic carcinoma (after FNAC). Intraoperative pathological examinations of frozen sections would facilitate surgical strategy-related decisions.

\section{Disclosure Statement}

The authors declare no conflict of interest.

\section{References}

-1 Kasamatsu A, Shiiba M, Nakashima D, Shimada K, Higo M, Ishigami T, Ishige S, Ogawara K, Tanzawa H, Uzawa K: Epithelioid myoepithelioma of the hard palate. Oral Maxillofac Surg 2013;17:63-66.

$>2$ Isogai R, Kawada A, Ueno K, Aragane Y, Tezuka T: Myoepithelioma possibly originating from the accessory parotid gland. Dermatology 2004;208:74-78.

-3 Xie L, Zhang D, Lu MM, Gao BM: Minimally invasive endoscopic-assisted resection of benign tumors in the accessory parotid gland: 5 case studies. Head Neck 2012;34:1194-1197.

4 Kawashima Y, Kobayashi D, Ishikawa N, Kishimoto S: A case of myoepithelioma arising in an accessory parotid gland. J Laryngol Otol 2002;116:474-476.

-5 Sun G, Hu Q, Tang E, Yang X, Huang X: Diagnosis and treatment of accessory parotid-gland tumors. J Oral Maxillofac Surg 2009;67:1520-1523.

6 Wang S, Shi H, Wang L, Yu Q: Myoepithelioma of the parotid gland: CT imaging findings. AJNR Am J Neuroradiol 2008;29:1372-1375.

7 Ikeda K, Katoh T, Ha-Kawa SK, Iwai H, Yamashita T, Tanaka Y: The usefulness of MR in establishing the diagnosis of parotid pleomorphic adenoma. AJNR Am J Neuroradiol 1996;17:555-559.

-8 Yabuuchi H, Matsuo Y, Kamitani T, Setoguchi T, Okafuji T, Soeda H, Sakai S, Hatakenaka M, Nakashima T, Oda Y, Honda H: Parotid gland tumors: can addition of diffusion-weighted MR imaging to dynamic contrastenhanced MR imaging improve diagnostic accuracy in characterization? Radiology 2008;249:909-916.

\$ Ramdall RB, Cai G, Levine PH, Bhanote M, Garcia R, Cangiarella J: Fine-needle aspiration biopsy findings in epithelioid myoepithelioma of the parotid gland: a case report. Diagn Cytopathol 2006;34:776-779.

10 Das DK, Haji BE, Ahmed MS, Hossain MN: Myoepithelioma of the parotid gland initially diagnosed by fine needle aspiration cytology and immunocytochemistry: a case report. Acta Cytol 2005;49:65-70. 
Iguchi et al.: Epithelioid Myoepithelioma of the Accessory Parotid Gland: Pathological and Magnetic Resonance Imaging Findings
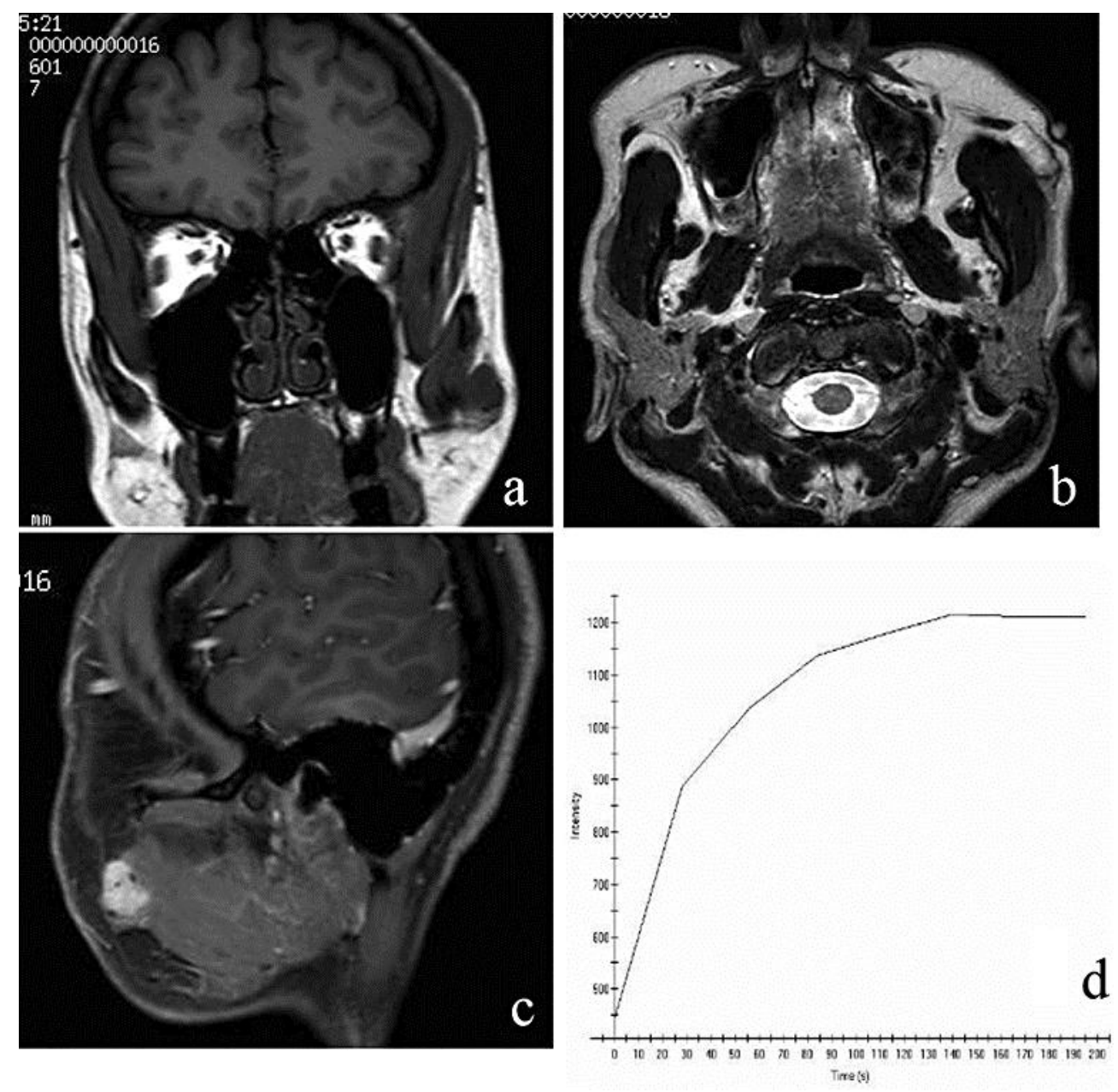

Fig. 1. MRI tumor findings. a Coronal T1-WI, b axial T2-WI, c sagittal contrast-enhanced T1-WI, and d time-signal intensity curve. The well-circumscribed and lobulated tumor was located anterior to the left parotid gland and on the outer layer of the masseter muscle. The tumor demonstrated isointensity on T1WIs and hyperintensity with peripheral hypointensity on T2-WIs, but was homogeneously well enhanced in contrast-enhanced T1-WIs. A time-signal intensity curve during a dynamic MRI study revealed a gradual enhancement pattern. 
Iguchi et al.: Epithelioid Myoepithelioma of the Accessory Parotid Gland: Pathological and Magnetic Resonance Imaging Findings
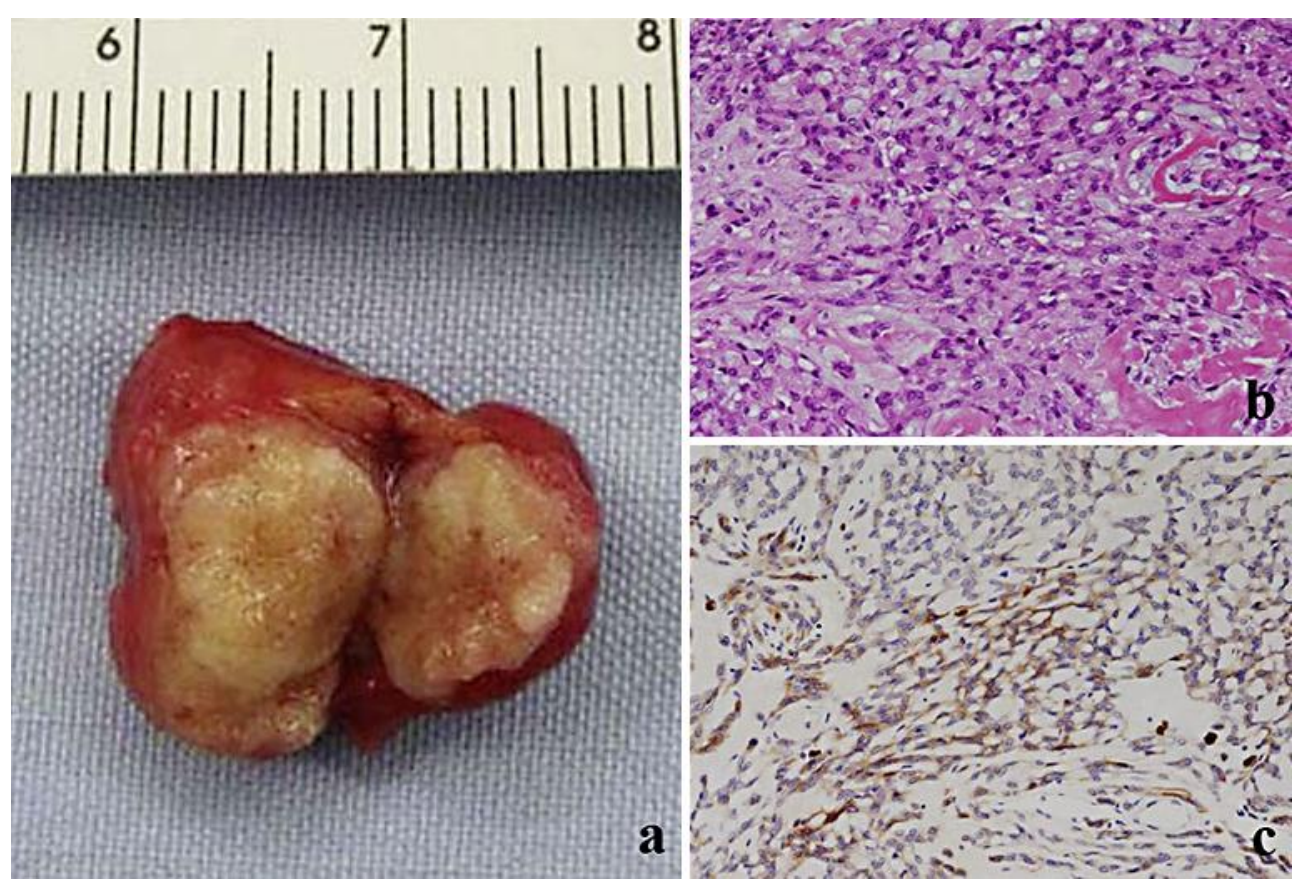

Fig. 2. a Gross appearance of the tumor, b microscopic appearance of the tumor (HE staining. $\times 200$ ), c immunohistochemical staining for calponin $(\times 200)$. The tumor was solid, lobular, and completely covered with a fibrous capsule. The neoplastic cells were arranged in an epithelial and mostly lattice-like structure with hyalinous and mucinous stroma. Neither cartilaginous myxoid stroma nor a glandular structure was observed. Immunohistochemical calponin staining was positive, indicating a myoepithelial nature. 